\title{
Research on the Design of Foldable Community Public Space Under the Concept of Micro-renewal
}

\author{
Jianing $\operatorname{Pan}^{1}$ Xiaojuan Hui ${ }^{1, *}$ \\ ${ }^{1}$ Nankai University Binhai College, Tianjin 300270, China \\ "Corresponding author. Email: teresahui@foxmail.com
}

\begin{abstract}
The accelerating process of urbanization in recent years has given rise to people's spiritual aesthetic needs, and the original infrastructure construction is not enough to meet the psychological needs of contemporary people. In recent years, urban micro-renewal design has been carried out in more cities, increasing their vitality. This paper analyzes and interprets the element of fold space on the basis of micro-renewal to explore the horizontal and vertical dimensions of design within a space. Cases and literature are used to explore the role of folded space in community public space. The current situation and reasonable design scheme of micro-renewal inside and outside China are also studied to formulate countermeasures in both theoretical and practical aspects. The folded spatial order and the visual effects of the scene are also taken into account to create a spatial superposition. Some of the usual micro-renewal approaches are employed, i.e. new creative spaces, "tree" spaces, color updates, material updates, etc. These are combined with the folding method to design a new scheme to revitalize the old community.
\end{abstract}

Keywords: Micro-renewal, Folding space, Community public space, Design.

\section{INTRODUCTION}

\subsection{Project Background}

In order to solve a series of problems caused by the rapid development of modern urbanization, designers in different fields began to adopt diverse ways to make micro-renewals. The problem of rapid population growth is also not to be underestimated. The world population will double by 2050 , and urbanization has become an inevitable trend.

Therefore, adopting appropriate micro-renewal strategy, rather than overall planning, contributes a lot to regional development. The existing community can be used as a pilot to improve the public space of the community with the help of micro-renewal, and then gradually promote the whole area. The concept of folding runs through the implementation plan and the overall design. In this case, folding means turning one part of an object over so that it blends with another. The development of the era has enriched the connotation of this term and made it applied to more fields. Thanks to the maturity of technology and modelling software, it has been applied in the design of architecture, landscape and product, and has brought about a great change to them, which enriches the diversity of design.

\subsection{Purpose and Significance}

The problems caused by shrinking areas of public space, rapid urbanization due to rapid population growth, and previously inadequate urban planning are all the more evident today. In order to restore the vitality of the city, micro-renewal is adopted. Chaos theory, folding sub-theory, fold space, and some conventional micro-renewal methods are employed to transform the community creatively.

\section{LITERATURE REVIEW}

\subsection{Micro-renewal}

\subsubsection{The Concept of Micro-renewal}

At present, the concept of micro-update in the development stage is still not perfect, so there is no widely accepted concept. Therefore, the author consults a lot of literatures concerning microrenewal. 
Micro-renewal is closely related to the protection of buildings, communities, and history and culture in cities, which can be proved in the following articles: The Research of Community Public Space Design from the View of "Micro-renewal" (Gong Rui, 2019), Research on the Strategy of Micro-renewal of Pedestrian Space in Old Communities (Duan Feixia, 2019), Research on Micro-renewal of Urban Community Public Space (Yang Lisheng, 2019), Research on the influencing factors of public participation in community micro-renewal (Zheng Huanhuan, 2019), and Research on micro-renewal evaluation system of Shanghai community public space (Zhong Xiao, 2020). However, it is worth noting that the previous research on micro-renewal should be used as a reference to take the essence and discard the dross.

\subsubsection{The Significance of Micro-renewal}

Micro-renewal aims to improve the public space of the community, enhance the vitality of the community, and promote the sustainable development of the community, which has become an inevitable trend of urban development. Such continuous and gradual approach is also important for everyday urban development. In addition to following the principle of sustainability, community micro-renewal should also maintain the unique features of the original old community and control the economic cost and scale. On this basis, a series of problems facing the community, such as transportation, aging infrastructure, cultural landscape, etc. can be addressed.

\subsection{The Application of Folding Space in Community Public Space}

\subsubsection{Research Theories Outside China}

\subsubsection{Deleuze and "The Fold"}

In 1986, postmodern philosopher Deleuze reinterpreted Leibniz's monad theory in his book "The Fold: Leibniz and the Baroque". Deleuze provides a new view to Leibniz's monad theory. He creatively proposed the continuously changing and diverse fold world, and believed that the space and time composed of the world were finally generated by the folding, unfolding and refolding of matter. These varied folds result in extremely complex selforganizing phenomena. (See "Figure 1")
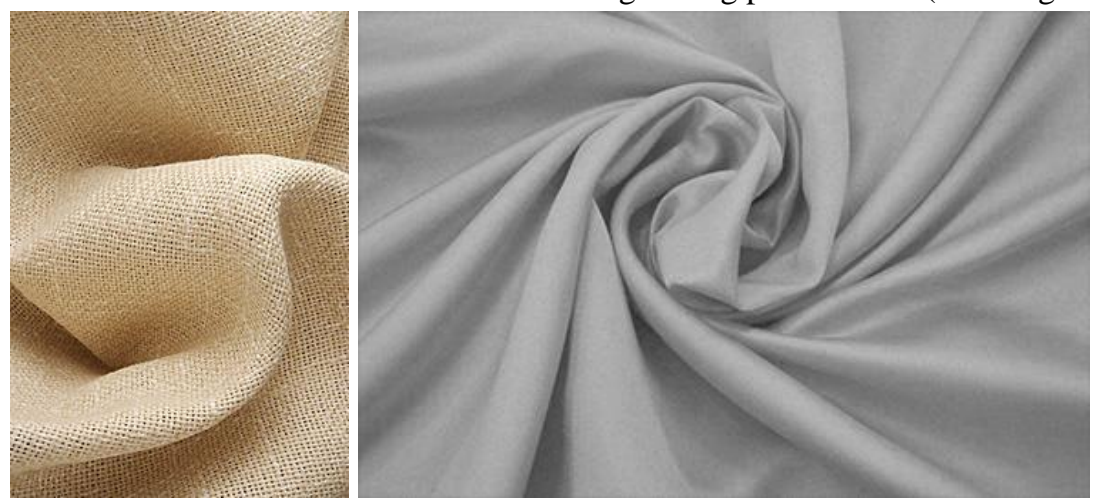

Figure 1 Schematic diagram of fold theory (Image source: Baidu Pictures).

The talk of theory is often connected with the matter of reality. Since the theory of fold did not provide an accurate definition of fold, fold was initially regarded as a folding wrinkle, such as the ubiquitous mountains, hills, ditches on river beaches, and the veins of plant leaves, which form mysterious and beautiful folds. (See "Figure 2") 


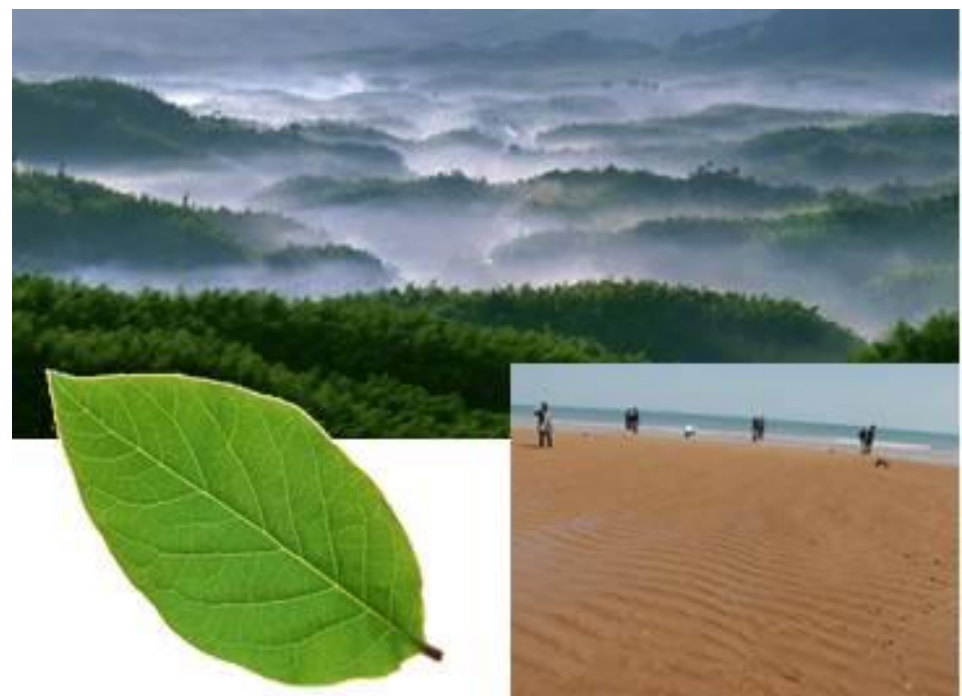

Figure 2 Schematic diagram of fold theory (Image source: Baidu Pictures).

All of these are microscopic folds. The modern world generally includes the natural world and the human world (specific to the city). The world, from the world map to the town map, is composed of cities and natural environment, thus forming the folding unit of city and nature rotation, and thus forming the current reality world. (See "Figure 3")

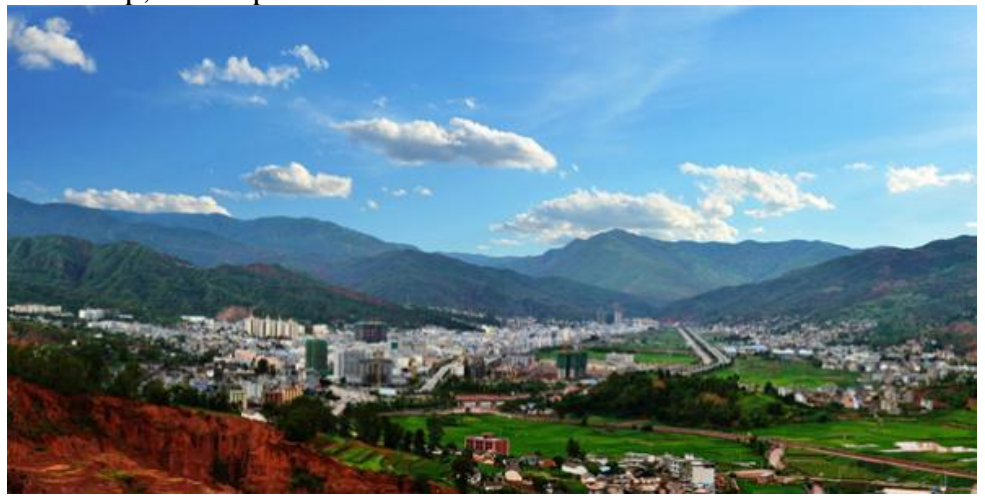

Figure 3 Schematic diagram of fold theory (Image source: Baidu Pictures).

On the philosophical level, the fold theory has a certain universality because it exists at all times and is infinite. Deleuze's fold theory is not material, but abstract and ideological. The fold involved in this theory can be subdivided continuously, for example, the types of people, the thoughts and personalities of different people will vary greatly and little, and these differences are all based on a certain point. As we all know, opinions of Hamlet vary from person to person, and Hamlet is the starting point. (See "Figure 4")
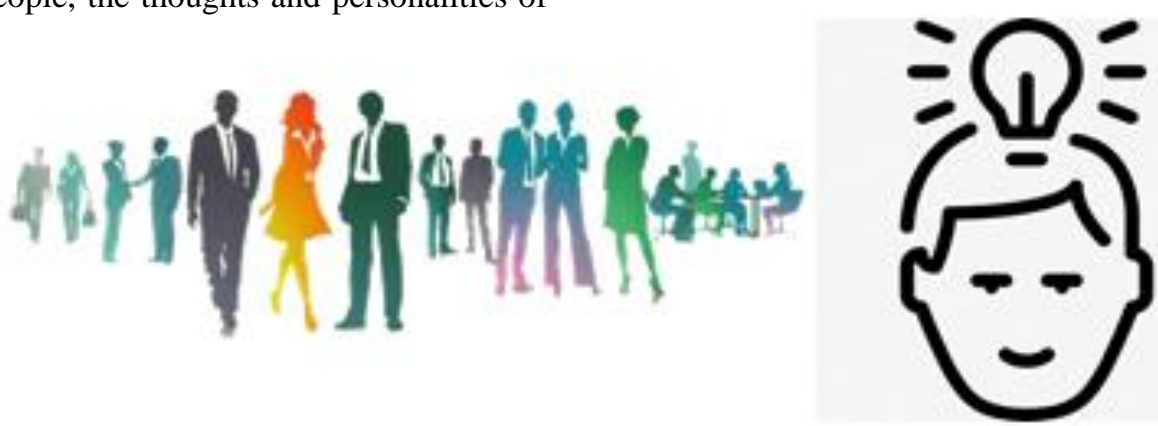

Figure 4 Schematic diagram of chaos theory (Image source: Baidu Pictures). 
The form of the design scheme is divided into artistic form and mental form according to the subjective and objective differences. Objects must first have artistic forms, and then they are perceived and transformed into mental forms. People's feeling of art comes from the perception and resonance of the form, so the products, buildings, landscapes and so on designed by designers should have fold, hierarchy in the external art form, and also give people the spiritual fold, that is, the effect of ups and downs, layers of impact of the mind.

\subsubsection{Chaos Theory}

Chaos theory is a method that combines qualitative thinking and quantitative analysis. It discusses the behavior of dynamic systems that cannot be explained and predicted by single data relations, but by whole continuous data relations.

Among chaos theories, catastrophe theory is most closely related to "folding" theory. The order and disorder, certainty and randomness, finite and infinite that chaos pursues make it reflect the state of real existence, which also leads to the transformation of people's traditional aesthetic ideas and the pursuit of infiltration and softening of architectural space form. (See "Figure 5")
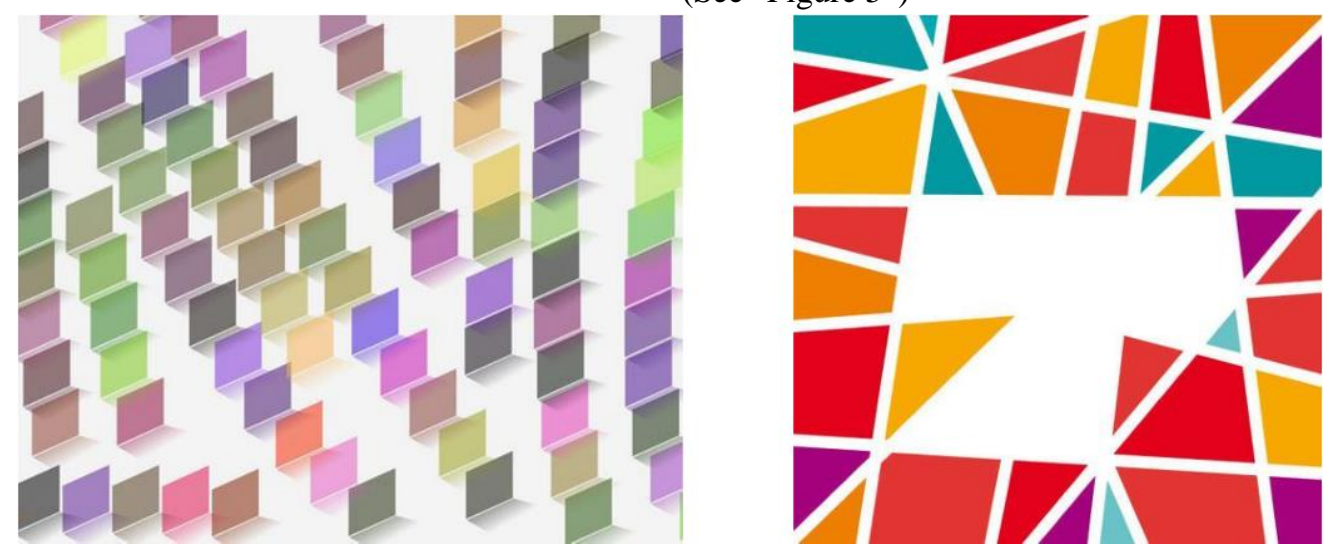

Figure 5 Schematic diagram of chaos theory (Image source: Baidu Pictures).

Chaos theory has many different interfering factors in the formation of urban structure since ancient times, which are mainly generated through the combination of natural evolution and some selfplanning behaviors of human beings, such as natural terrain, landscape, climate, human thoughts and population size, etc. This is a dynamic process that progresses over time.

Chaos theory is stable as a whole, but uncertain locally. In the process of urban change, one link can lead to subtle differences, which can lead to radically different results. This is similar to the "butterfly effect", which makes it difficult for urban planners to accurately assess their long-term impact on urban planning.

At present, the urban planning pursues simplicity to avoid the random influence brought by the uncertainty of chaos, which is very lack of freedom. The unpredictability of chaos theory should be applied to urban design. For example, in the construction of modern new countryside, straight roads replace the original natural winding path, and the original mode will be more dynamic than the current way, and it will also bring more sense of belonging tolocal residents, and increase the human interest. Therefore, designers should accept such chaos, make reasonable use of chaos to carry out urban construction, and avoid design that's too neat. (See "Figure 6") 

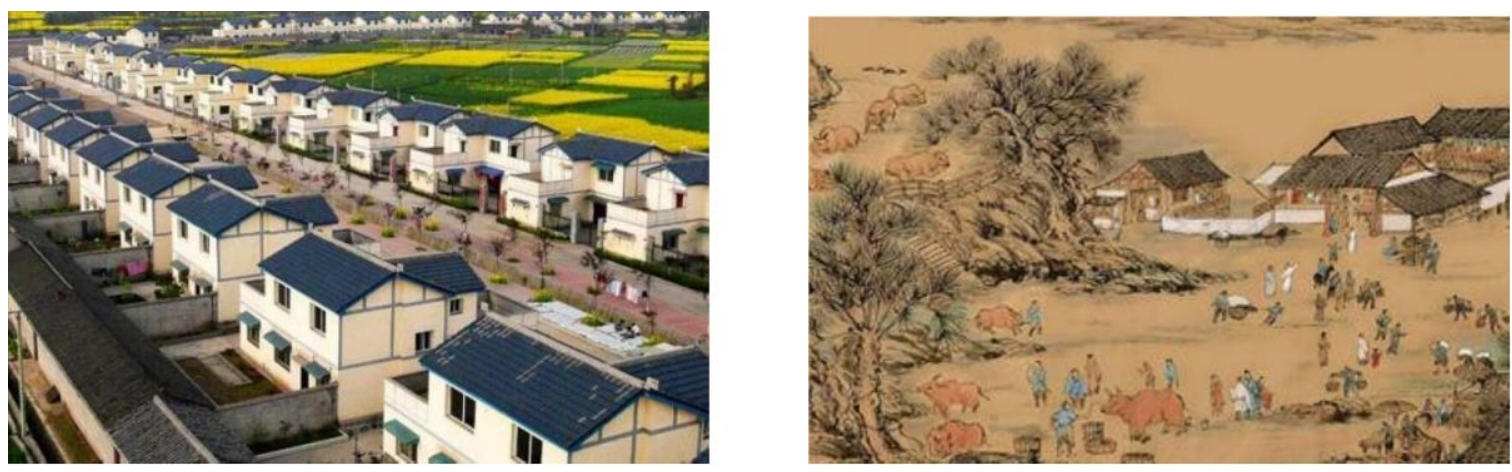

Figure 6 Countryside before and after micro-renewal (Image source: Baidu Pictures).

\section{RESEARCH ON FOLDING CHARACTERISTICS UNDER THE CONCEPT OF MICRO-RENEWAL}

\subsection{Design Strategy of Folding Space}

Strategy is a special problem-solving method and a dynamic process, which requires continuous exploration of the form of the block and its corresponding functions, and clarifying the complex relationship between social status, culture and people. The search for the corresponding strategy is a process of discovering the potential of the area, and eventually what is found can be integrated and used to improve the design and add new vitality.

Designers should explore the space and carefully observe the overall composition and formation of the space. One of the most important elements of a space is the central path, with which the relationship between horizontal and vertical can be clarified. This can better guide the visual senses of other experiencers as well as their own actions, so that experiencers can better experience each space that's specially designed. Therefore, the choice of path is critical to micro-renewal.

\subsubsection{The Tenerife Theatre Centre}

The theatrical arts centre uses the urban landscape as the backdrop for its stage. The interior roof terrace is folded from a three-dimensional wooden platform, and the stage background frame redefines the space between the city and the building, promoting the interactive relationship between them. (See "Figure 7")

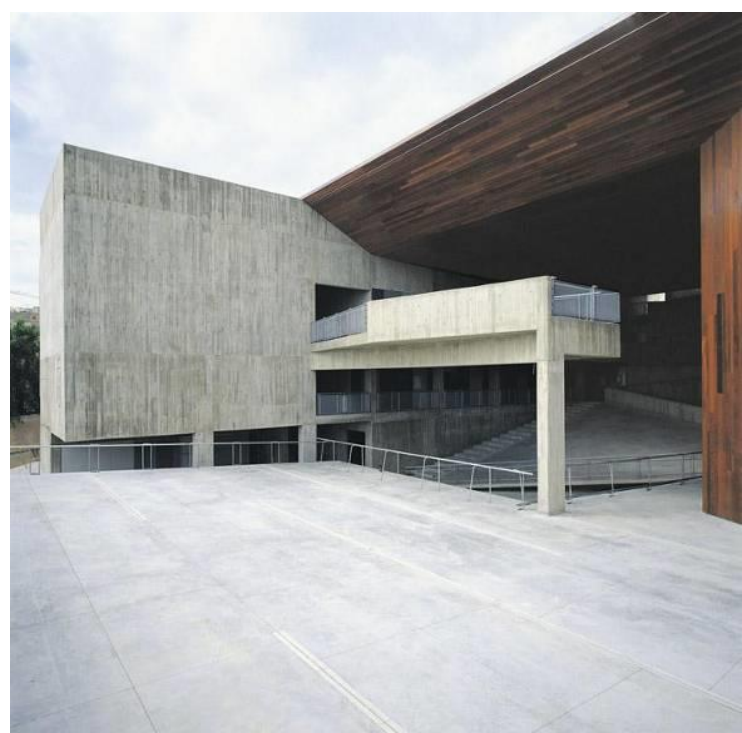

Figure 7 The Tenerife Theatre Centre (Image source: https://www.gooood.cn/).

The art center features a variety of sloping planes, a pitched roof that functions as an open-air auditorium, and sloping zigzag ramps that act as pedestrian traffic streamlines. This architectural space can be completely transformed into a public and open space for people to perform. The audience ramps, concrete platforms, and ground completely dissipate 
the boundaries between the actors and the audience.

(See "Figure 8", "Figure 9")

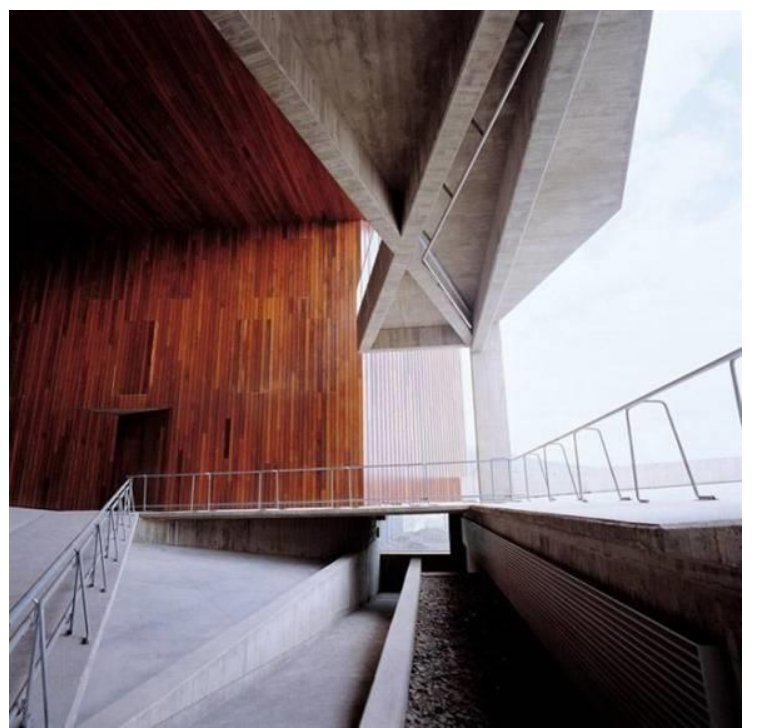

Figure 8 The Tenerife Theatre Centre (Image source: https://www.gooood.cn/).

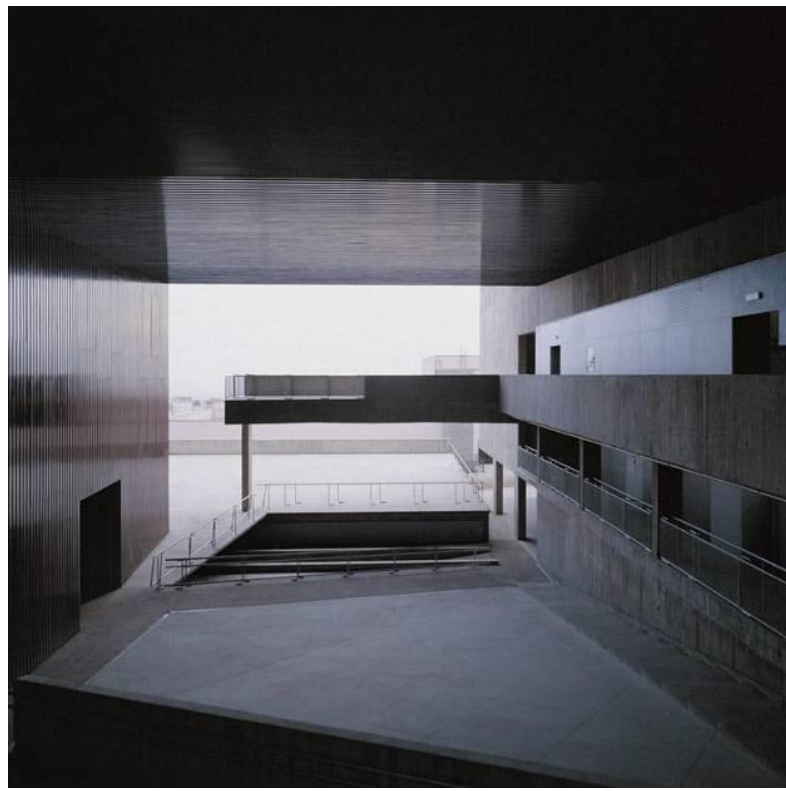

Figure 9 The Tenerife Theatre Centre (Image source: https://www.gooood.cn/).

\subsection{Visual Richness in Space}

\subsection{1 "Tree" Space}

The "tree" space is one of the more important design units in the community. The old community may be faced with problems such as scarce tree species and poor combination effect, so new tree species can be added to form different combinations, so that the public space in the community, especially some original blank space, can be more abundant.

\subsubsection{Creative Space}

The unique historical culture of the old community or its blending with the emerging culture is one of the important means to radiate the vitality of the community. Therefore, the community space can be divided according to its functions to create different cultural and creative spaces, such as featured landscape structures and wall painting. At the same time, originality should also be valued, because cookie-cutter design is not desirable. 


\subsubsection{Folding Space}

The use of vertical space is often overlooked. Poor planning of vertical spaces can lead to problems such as reduced lighting, air flow, and feelings of depression. However, if these possible problems can be solved, the richness of the spatial structure system of the whole community will be greatly enhanced.

\subsection{The Medium of Harmonious Coexistence Between Man and Space}

When the public space is a social medium, the most basic element is the material element, and the environmental designer should make a reasonable layout of the space. Whether it is urban public space, park public space or community public space, the elements that need to be involved are basically the same, such as color, material, site path, functional partition, plant configuration and so on.

All kinds of elements in the community public space should be connected in an orderly way, which requires a reasonable handling of the connection between people and space. In other words, connectivity is one of the most important elements in public space.
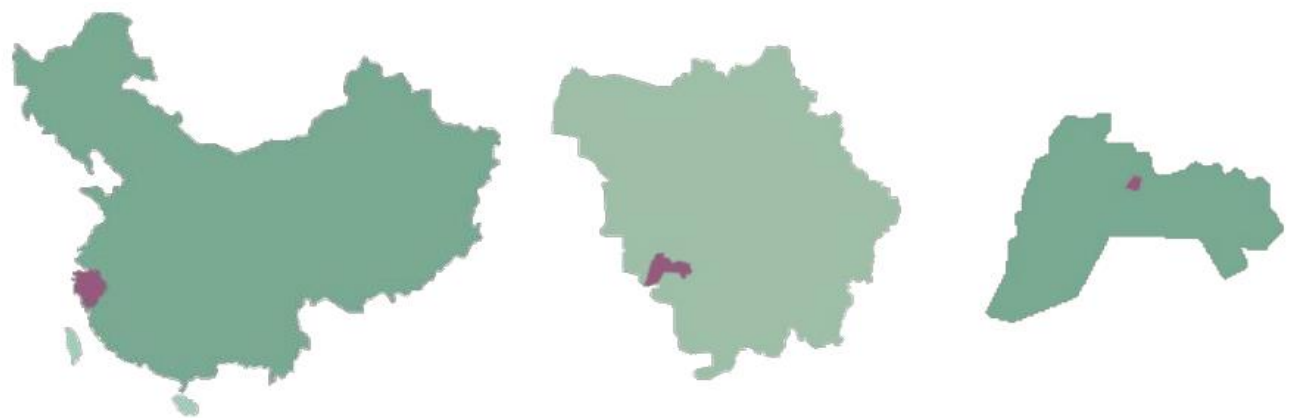

Figure $10 \quad$ Research object (Image credit: Author's own drawing).

\subsection{Field Investigation}

Main roads and residential buildings in the community are basically parking areas, as well as green belts, and there is basically no space for entertainment. The community is faced with problems such as too many vehicles, narrow activity space, lack of infrastructure and aging buildings, which need to be improved as soon as possible. (See "Figure 11") 


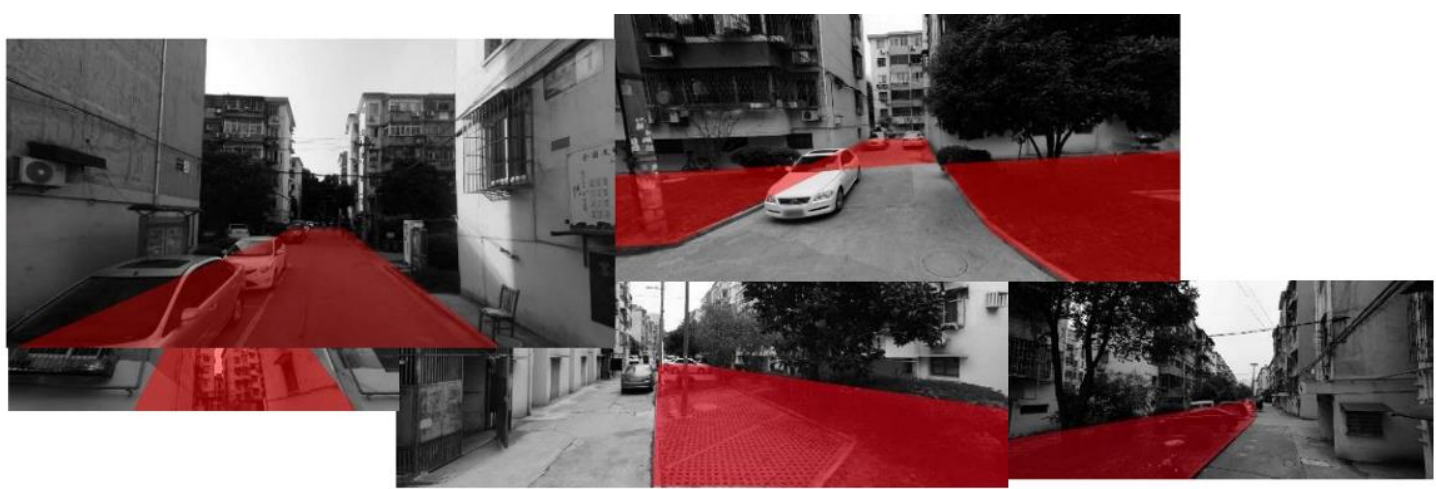

Figure 11 Analysis of site status (Image credit: Author's own drawing).

According to the site survey and some basic problems from the aging of the city, it is concluded that the community is faced with such problems as lack of vitality, small native space and no leisure space. Different strategies have been developed based on three questions: new creative spaces, new colors, new materials, and new local culture or celebrities. The concept of folded space is combined with fold theory, chaos theory and the form of creative space to carry out micro-renewal. In response to the lack of leisure space, playgrounds, walkways and "tree" spaces have been added. Of course, these strategies are complementary and indispensable.
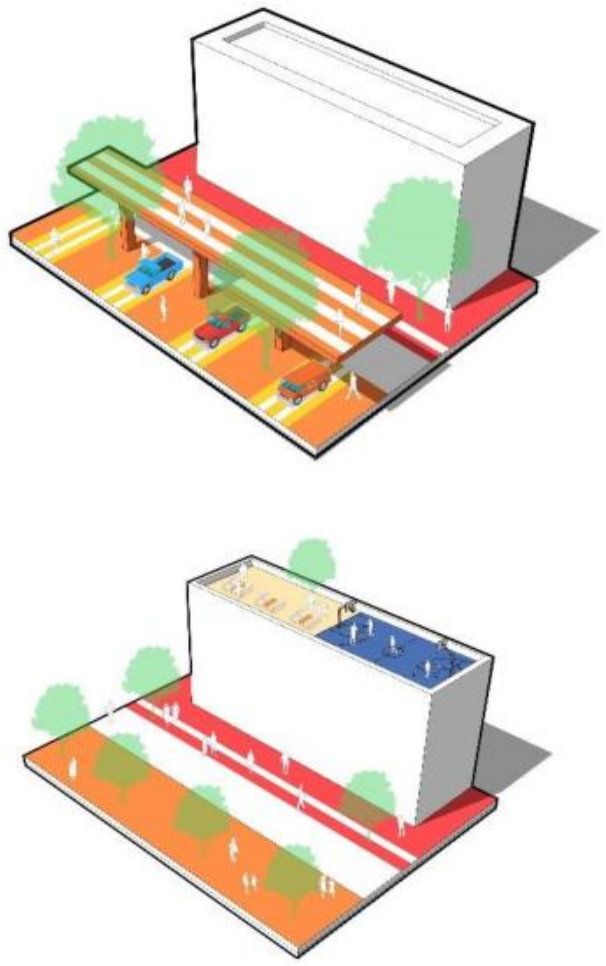

By analyzing the characteristics of the word "folding" and combining with local culture, landscape structures or mobile devices with local characteristics are made. In addition, simple folding devices can be adopted to increase the area of the space for leisure and entertainment. Some basic community micro-renewal solutions have been adopted, such as color updating, creative space deepening, "tree" space design, etc. These design methods can also be used in combination with folding principles in certain circumstances to create different effects.

The design concept can be seen in "Figure 12".

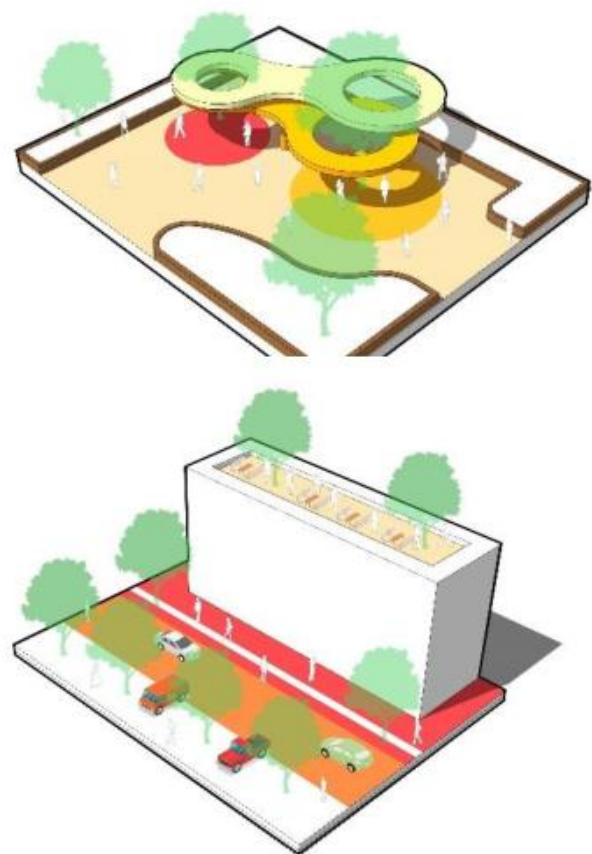

Figure 12 Improvement strategy of the community (Image credit: Author's own drawing). 


\subsection{Implementation Process}

Different color indicators have been applied to the neighbourhood, such as white and orange stripes for the updated parking areas, red and orange for the pedestrian space, beige for the open space, and white and orange stripes for the elevated walkways. In this way, the functional division of the community is more obvious. (See "Figure 13")

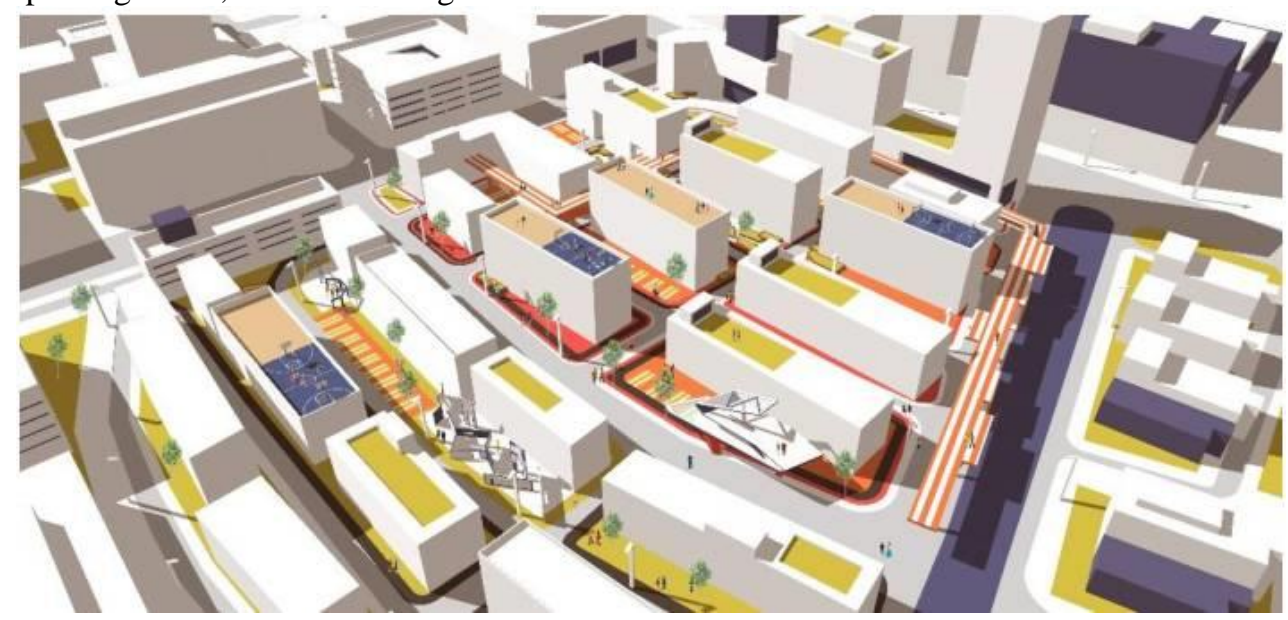

Figure 13 An aerial view of the community (Image credit: Author's own drawing).

The whole community is divided into three parts, and the fold theory, chaos theory and the form of creative space are used to improve the whole

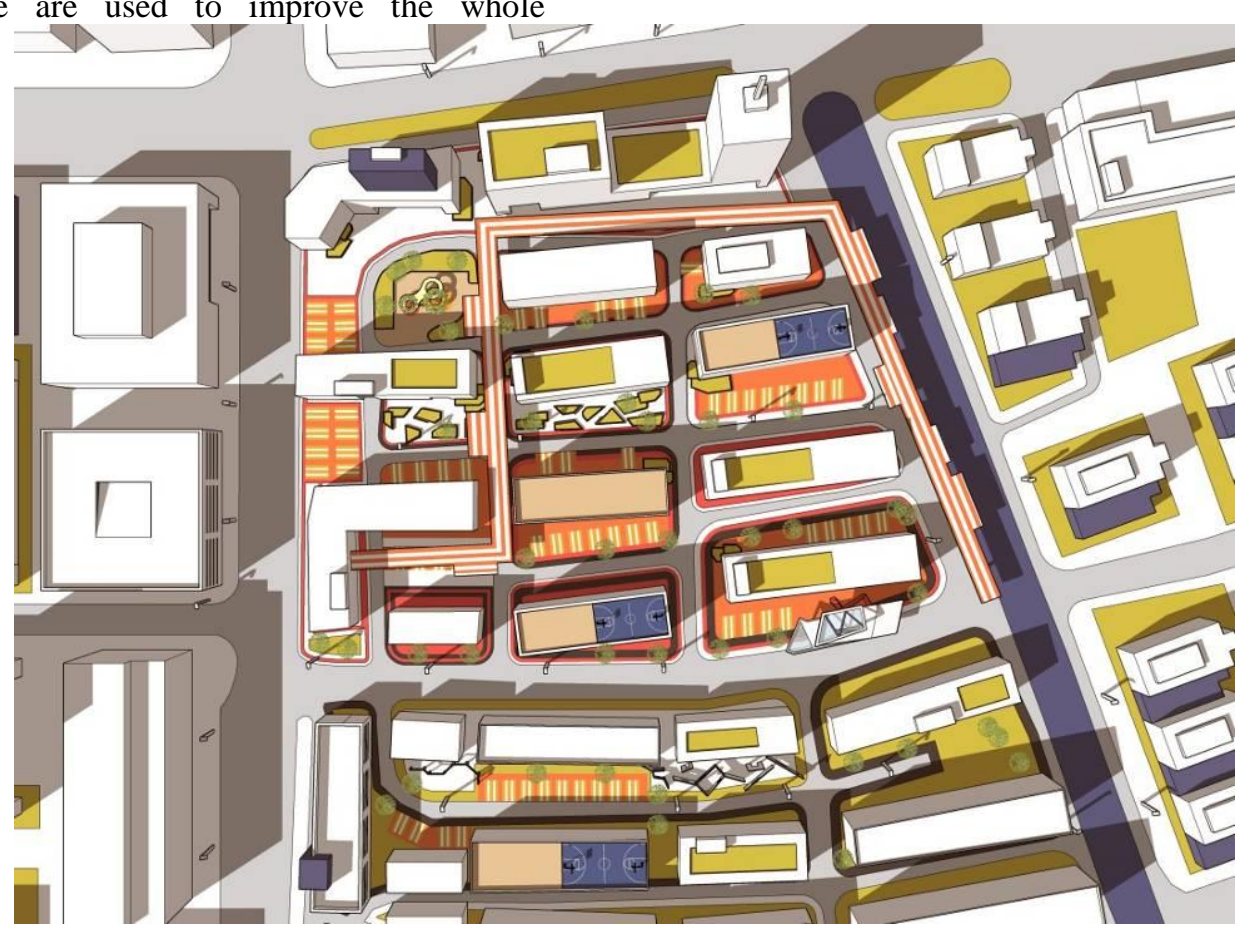

community, and color is used to achieve the harmony of the whole community. (See "Figure 14")

Figure 14 The floor plan of the community (Image credit: Author's own drawing).

The infrastructure has been completely refurbished, existing parking Spaces, plants and so on have been completely reconfigured. For example, the parking lot changed from the original grey cement to the red and orange stripes, which is more unique. 


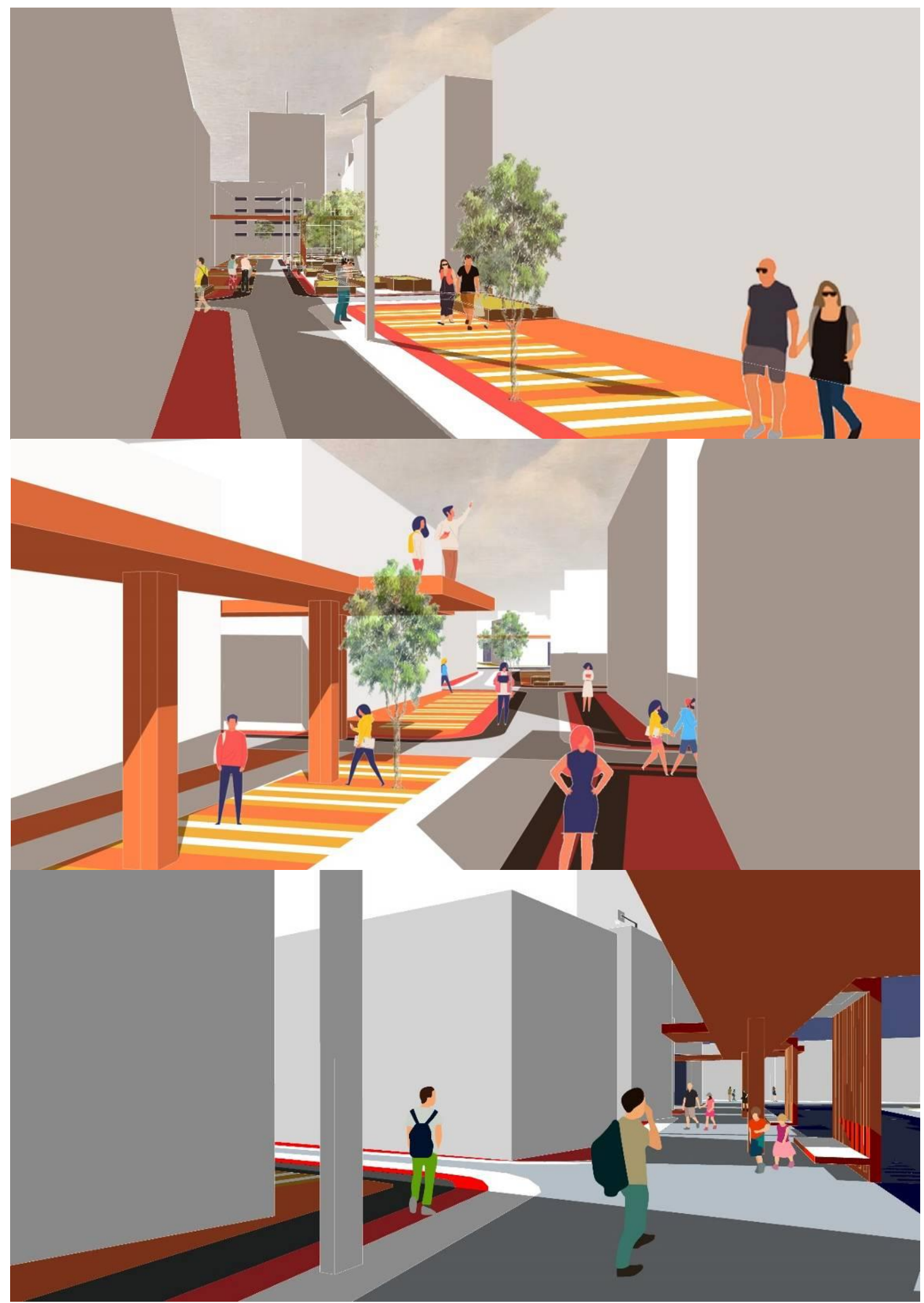

Figure 15 The internal transformation of the community (Image credit: Author's own drawing).

At the same time, several groups of folding devices designed with different theories were added to expand the horizontal and vertical visual richness respectively, and to add more area to the limited space. 
In order to enhance the local characteristics of the community, some special literati culture will be integrated into the design process to enhance the charm of hometown. (See "Figure 16", "Figure 17", "Figure 18")
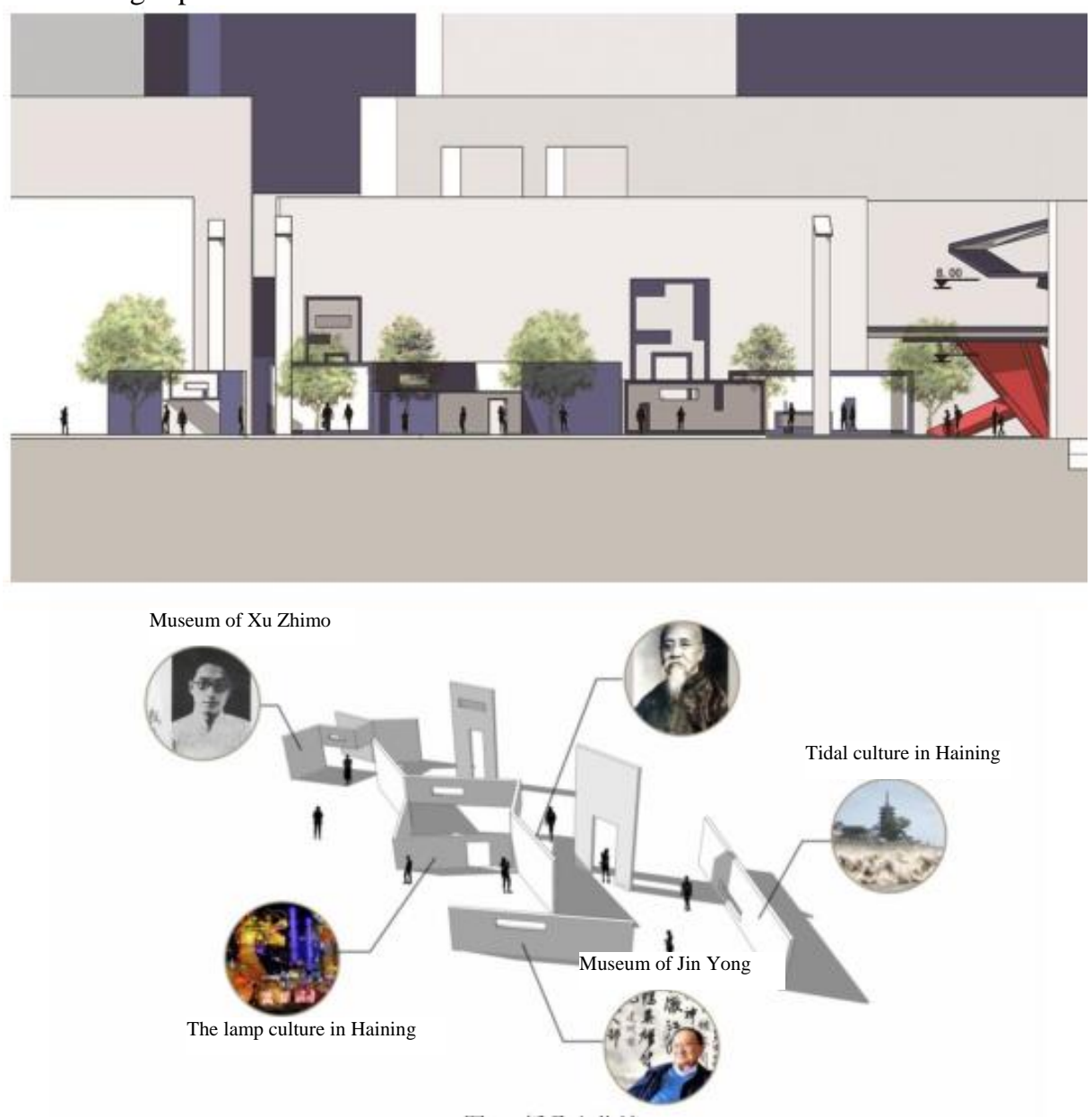

Figure 16 Folding Culture Wall (Image credit: Author's own drawing).

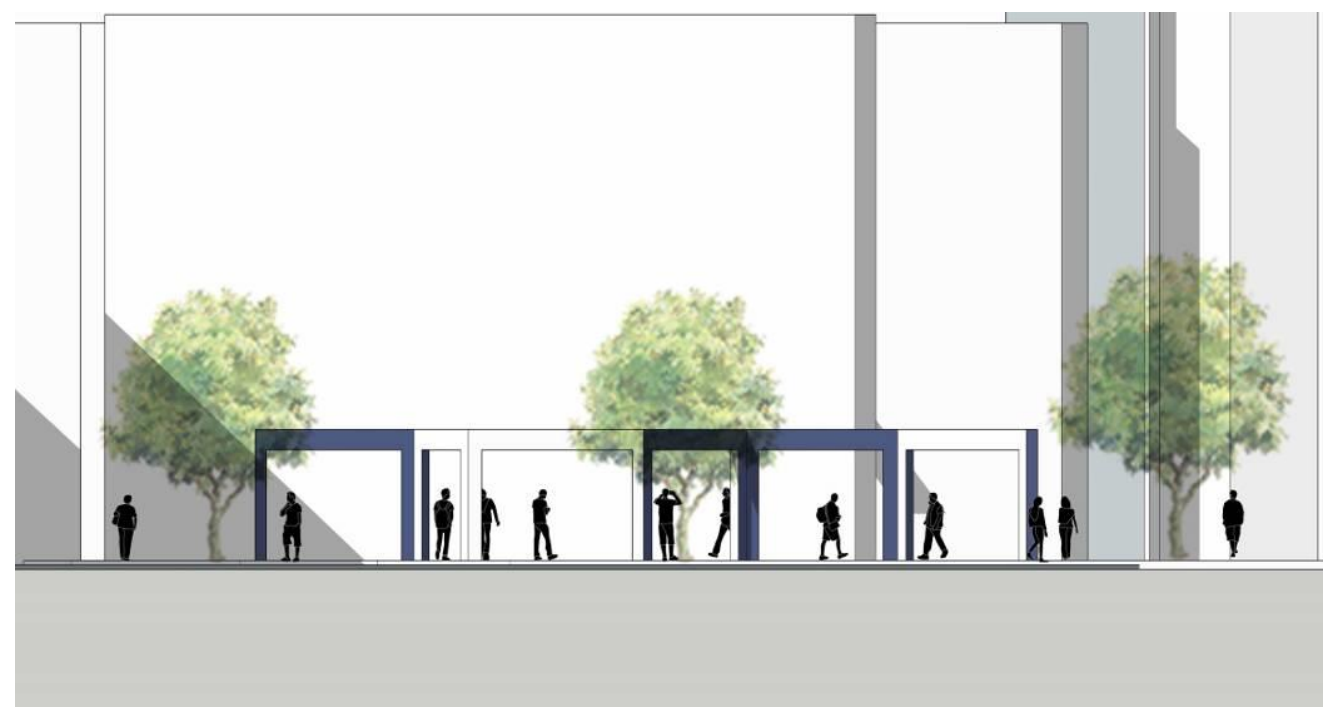

Figure 17 Folding Interactive Wall (Image credit: Author's own drawing). 


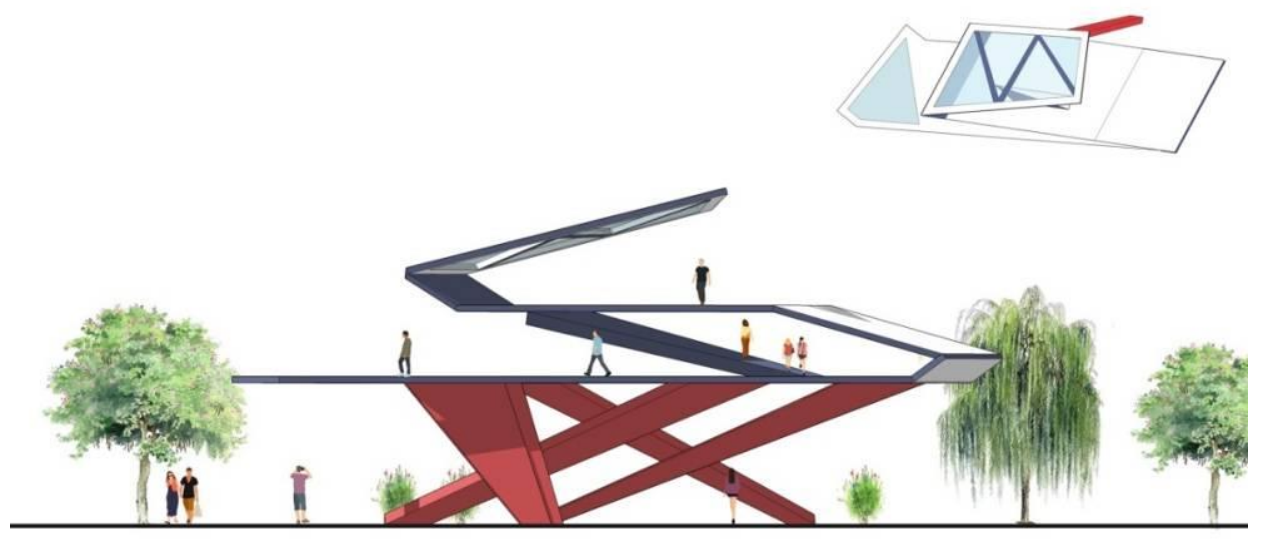

Figure 18 Folding movable device (Image credit: Author's own drawing).

\section{CONCLUSION}

As for the micro-renewal of the old community, the first is to read a lot of literature to understand the micro-renewal and the micro-renewal strategies and methods, and carry out a new design through the combination of micro-renewal and application methods. The extension of the space and the resulting visual richness should be exploited for design analysis in order to realize the folding concept's contribution to the renewal of the community. Conventional renewal methods should also be used to further enrich the community's activity space. The randomness and freedom brought by chaos theory, and the infinite hierarchy of fold theory, have a great influence on the transformation of the community and the change of the whole society. However, due to the author's limited energy and lack of research ability, there are still many deficiencies in the proposed scheme.

Therefore, the concept of micro-renewal is supposed to be improved in the future to truly serve the society with design. Many problems arising from urban development can also be deeply studied to make a contribution to the design of China.

\section{AUTHORS' CONTRIBUTIONS}

Xiaojuan Hui contributed to Cconceptualization, whiting-review, and supervision, and Jianing Pan is responsible for compiling, research and summary of data, and plan design.

\section{REFERENCES}

[1] Cai Mengxuan. Folding: S-M A. O. Space Design Practice [D]. Soochow University, 2016. (in Chinese)
[2] Fu Yirong. Folding Space: Research for Form and Design Strategy of Multi-level Space [D]. Tongji University, 2006. (in Chinese)

[3] Yang Bin. The Research of Non-linear Form Design in Architectural Space [D]. Hunan Normal University, 2016.

[4] Cai Mengxuan. Folding: S-M A. O. Space Design Practice [D]. Soochow University, 2016. (in Chinese)

[5] Gong Rui. The Research of Community Public Space Design from the View of "Microrenewal" [D]. East China Normal University, 2019. (in Chinese)

[6] Wei Na. Research on the Public Space as Social Media [D]. Jiangnan University, 2018. (in Chinese)

[7] Duan Feixia. Research on the Strategy of Microrenewal of Pedestrian Space in Old Communities [D]. Hefei University of Technology, 2019. (in Chinese)

[8] Ding Yiwen. Research on Micro-update Design of Community Public Space [D]. Jilin Architecture University, 2019. (in Chinese)

[9] Song Ruochen. Research on Design Strategy of Public Space Micro-renewal in Old Urban Communities [D]. Harbin Institute of Technology 2019. (in Chinese)

[10] Pang Guoyu. The Pattern of Non-Governmental Organizations' Intervention into Urban Community Planning [D]. Zhejiang University, 2017. (in Chinese) 
[11] Fu Yiran. Architects Participate in Community Micro-renewal [D]. Qingdao Technological University, 2019. (in Chinese)

[12] Hu Xianjun. The Design Strategies Research on Spatial Micro-renovation of Lingnan Old City District Based on Climate Response [D]. South China University of Technology 2019. (in Chinese)

[13] Deng Shichao. Sustainable Regeneration Strategy Research for Improving the MicroClimate of the Urban Block [D]. Hubei University of Technology, 2014. (in Chinese)

[14] Xiong Huihua. Research on Strategies of Folding Theory in Landscape Architecture Design [D]. Hunan University, 2015. (in Chinese)

[15] Zhang W, Jia R. The creation of public nature in community public space design [C]. The 2014 Pacific-Asia Workshop on Computer Science in Industrial Application (CSIA 2014). 0.

[16] Jia Y. The construction of a public space model: the Community Public

[17] Jia Y. The construction of a public space model: the Community Public Life Channel[C]// 2012.

[18] Wei ZHANG, Ru, JIA. The Creation of Public Nature in Community Public Space Design [J]. International Technology Management, 2014(11):54-56. 